\title{
XXXI. Über die Krystallform des Britholiths.
}

\author{
Von \\ O. B. Böggild in Kopenhagen.
}

(Mit 2 Textfiguren.)

Der Britholith, der bisher nur bei Naujakasik im Julianehaab District, Grönland, gefunden worden ist, wurde im Jahre 1899 von C. Winther beschrieben 1). Die Krystallform wurde als rhombisch bestimmt mit dem Axenverhältnis:

$$
a: b: c=0,620: 1: 0,423 \text {. }
$$

Die Krystalle, die von den Flächen (010), (110), (130), (021) und (111) begrenzt werden, sind ausgeprägt pseudohexagonal und außerdem stark nach (110) verzwillingt, wobei jedes wexagonale* Individuum aus sechs rhombischen Sectoren aufgebaut ist. Der optische Axenwinkel ist klein und nicht genau meßbar.

Dies sind die wesentlichen Resultate der Untersuchungen von Winther; mehrere Einzelheiten werde ich im folgenden erwähnen.

Da ich bei Durchmusterung der Auswahl von Britholithmaterial im mineralogischen Museum in Kopenhagen unter anderem einige ganz kleine und stark glänzende Krystalle fand, die für eine goniometrische Untersuchung sehr ausgezeichnet zu sein schienen, unternahm ich eine solche und bin ich dabei zu dem Resultat gekommen, daB es keinen Grund gibt, nach welchem man den Britholith als rhombisch annehmen muß; das Mineral ist ganz sicher hexagonal mit optischen Anomalien, die übrigens sebr schwach hervortretend sind.

Wie bei Winther nachgewiesen, sind die gewöhnlichen Krystalle in geometrischer Beziehung außerordentlich schlecht ausgebildet. Dies beruht wohl nicht soviel darauf, daß die Flächen sehr uneben sind, sondern vielmehr auf dem Umstand, daß der Krystall im ganzen aus vielen hypo-

1) Meddelelser om Grönland 84, 190. Ausz. diese Zeitschr. 34, 682. 
parallelen Individuen zusammengesetzt ist, und bei diesem Aufbau zeigt es sich, daß die Prismenflächen nicht allein in der Prismenzone selbst aus ihrer Stellung abgelenkt sind, sondern auch, und gerade ebensoviel, außerhalb der Zone gedreht sind. Das letztere Verhältnis ist sehr wichtig, weil es sich nicht durch $Z$ willingsbildung in rhombischen Krystallen erklären läßt und dabei direct zeigt, daß der Krystallbau gestört ist und daß man also nicht zuviel auf die Unregelmäßigkeiten in den Prismenwinkeln geben darf.

Unter den zahlreichen Werten der Kantenwinkel in der Prismenzone hat Winther zwei gefunden, die oft wiederkehrten, nämlich $28^{0} 11^{\prime}$ und 3002' (die Werte für (010):(130) und (130):(110)); wenn der Krystall hexagonal ist, so ist der letzte Wert natürlich nicht so merkwürdig; daß der Wert von $28^{\circ} 11^{\prime}$ oft wiederkehrt, muß meiner Erfahrung nach auf einem Zufall beruhen. Ferner hat Winther, jedoch nur in einem Falle, die drei Winkel $34^{0} 43^{\prime}, 30^{0} 7^{\prime}$ und $28^{\circ} 5^{\prime}$ in dieser Reihenfolge gefunden; von diesen sollte der erste der Wert für (100):(1.10) (theoretisch 31047') sein, die letzten die beiden obengenannten. Daß man zwischen so vielen verschiedenen Werten drei nacheinander finden kann, die einigermaßen mit drei gegebenen übereinstimmen, beweist eigentlich nicht viel. Auch aus den Winkeln (021):(010) und (111):(110) läßt sich nicht viel schließen; die von Winther gemessenen Werte sind $49^{\circ} 48^{\prime}$ und ca. $51^{0}$ (theoretisch $\left.51^{0} 16^{\prime}\right)$; man erhält nämlich in den Winkeln zwischen den anscheinenden Pyramiden- und Prismenflächen ebenso große Variationen wie in den Winkeln in der Prismenzone, soda $B$ man auch hier leicht ein Paar wird aussuchen können, die einigermaßen mit den berechneten stimmen.

Die von mir im Anfang erwähnten ganz kleinen Krystalle (Durchschnittsgröße ungefähr $\frac{1}{2} \mathrm{~mm}$ ) haben bei der goniometrischen Untersuchung ein etwas anderes Bild ergeben als die gewöhnlichen Krystalle; einige von den kleinen Krystallen geben wohl auch recht abweichende Werte (doch ist die

\begin{tabular}{|c|c|c|c|c|}
\hline & \multicolumn{2}{|c|}{ Krystall I } & \multicolumn{2}{|c|}{ Krystall II } \\
\hline & $\varphi$ & $\varrho$ & $\varphi$ & $\varrho$ \\
\hline$m_{1}$ & $0^{0} \quad 0^{\prime}$ & $90^{0} 0^{\prime}$ & $0^{0} \quad 0^{\prime}$ & $89043^{\prime}$ \\
\hline$m_{2}$ & 5955 & 903 & 604 & 8943 \\
\hline$m_{3}$ & $119 \quad 54$ & 8958 & 1206 & $90 \quad 18$ \\
\hline$m_{4}$ & -17955 & 8958 & 17932 & 8945 \\
\hline$m_{5}$ & -1204 & 8957 & -12030 & 8931 \\
\hline$m_{6}$ & $-59 \quad 57$ & 8955 & -607 & 8950 \\
\hline$a_{1}$ & 3014 & 902 & - & - \\
\hline$a_{4}$ & $\begin{array}{ll}-150 & 0\end{array}$ & 8958 & - & - \\
\hline$p_{1}$ & -138 & 4129 & 031 & $40 \quad 7$ \\
\hline$p_{2}$ & 5657 & 4030 & 6030 & 3959 \\
\hline$p_{3}$ & 11827 & $39 \quad 49$ & 12026 & 3958 \\
\hline$p_{4}$ & - & - & 1792 & 3954 \\
\hline$p_{6}$ & - & - & -5952 & 3940 \\
\hline
\end{tabular}


Abweichung von den hexagonalen Werten selten mehr als 10); es gibt aber auch einige, die wesentlich genauer sind. Auf S. 431 sind die Positionswerte für die zwei besten Krystalle aufgeführt; die hexagonalen Formen sind: $m(10 \bar{T} 0), a(11 \overline{2} 0)$ und $p(10 \bar{T} 1)$.

Die vorstehenden Werte der $m$-Flächen bei Krystall I zeigen ganz deutlich, daß der Krystall hexagonal ist, oder, wenn er rhombisch sein sollte, doch einen Prismenwinkel besitzen müßte, der nur wenige Minuten von $60^{\circ}$ abweicht. Die Winkel an Krystall II sind zwar mehr abweichend; doch zeigt der Umstand, daß die $g$-Werte ebenso ungenau sind wie die $\varphi$-Werte, daß die Ungenauigkeit in Stürungen des Krystallbaues seinen Grund hat. Die Werte für die $p$-Flächen sind bei Krystall I ganz unmöglich zu gebrauchen, während sie an Krystall II recht gut sind; aus den fünf Werten für $\varrho$ erbält man den Mittelwert $39^{\circ} 55 \frac{1}{2}^{\prime}$, woraus das Axenverhältnis hervorgeht:

$$
c=0,7247 \text {. }
$$

Die gewöhnliche Form der Krystalle, die übrigens mit derjenigen des Apatits eine sehr große Ähnlichkeit hat mit der Ausnahme, daß die Basis bei dem Britholith niemals vorkommt, ist in Fig. 2 wiedergegeben.

Der optische Aufbau der Krystalle ist von Winther recht ausführlich beschrieben worden. Nach den Beobachtungen, die ich gemacht habe, gibt

Fig. 1.

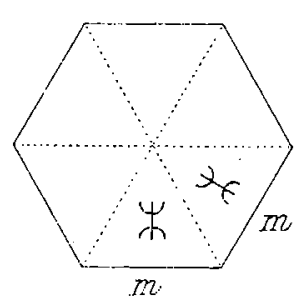

Fig. 2.

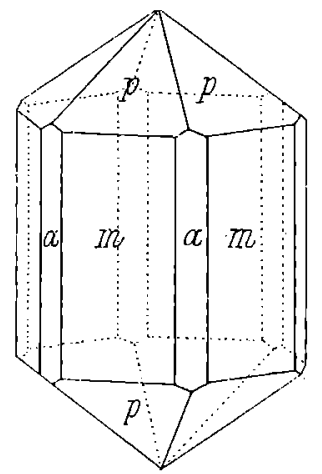

es jedoch viele Krystalle, die gar keine Anomalien zeigen, sich also vollkommen hexagonal verhalten, indem sie bei parallelem Licht in allen Stellungen vollkommen dunkel sind, in Schliffen nach der Basis und bei convergentem Licht vollkommen einaxig. Andere Krystalle zeigen optische Anomalien, die jedoch so schwach hervortretend sind, daß sie kaum ohne eingeschaltetes Gypsblättchen (Rot erster Ordnung) sichtbar werden; für die Beobachtung bei parallelem Licht wirkt der Umstand sehr störend, daß viele Partien wegen der mit dem Hauptindividuum nicht parallelen Stellung recht deutliche Interferenzfarben zeigen. Sieht man davon ab, so besteht 
der anomale Krystall in basischen Querschnitten aus sechs Sectoren, deren Grenzlinien vom Centrum nach den Ecken des Hexagons gehen, wie aus der schematischen Fig. $1 \mathrm{zu}$ ersehen ist. Die optische Axenebene steht in jedem Sector senkrecht zur Hexagonkante; der Axenwinkel ist, wie früher erwähnt, sehr klein und läßt sich höchstens zu $10^{\circ}$ schätzen.

Es entsteht jetzt die Frage, ob man nicht etwa aus den optischen Verhältnissen allein schließen könnte, daß das Mineral rhombisch ist; es gibt ja doch Mineralien, z. B. Boracit, die geometrisch gar keine Abweichungen von der höher symmetrischen Krystallform zeigen und die doch von den meisten des optischen Aufbaues wegen zu dem weniger symmetrischen Krystallsystem gerechnet werden. Es existiert wohl sicher kein einzelnes Unterscheidungsmerkmal zwischen mimetisch gebauten und optisch anomalen Krystallen, und die verschiedenen Autoren gehen auch in ihrem Urteil darüber sehr weit auseinander; bekanntlich deutet Mallard ja sozusagen jede optische Anomalie als von Zwillingsbau herrührend, während Klein, Ben Saude u. a. das meiste für nur optische Anomalien halten, was nicht wegen der geometrischen Eigenschaften zu einer niedrigeren Symmetrie gerechnet werden muß. Im allgemeinen weichen wohl jetzt die Anschauungen der verschiedenen Autoren nicht so sehr von einander $a b$, wenn es sich um die mehr typischen Fälle dreht, während es stets eine große Menge von Substanzen gibt, die sehr schwierig in die eine oder andere Classe einzuordnen sind. Ich werde im folgenden die wichtigsten Unterscheidungsmerkmale zwischen mimetischen und optisch anomalen Krystallen, soweit sie sich in den Dünnschliffen allein zeigen, zusammenstellen 1 ).

I. Die anomalen Krystalle sind im allgemeinen in optischer Beziehung inhomogen, indem die optischen Constanten von einer Stelle zur anderen variieren können; wie bekannt, wechseln nicht allein die Auslöschungsrichtungen und die Stärke der Doppelbrechung, sondern auch die Zahl der optischen Axen, das Vorzeichen usw. Dagegen ist jedes einzelne Individuum eines mimetischen Krystalls ebenso homogen wie ein gewöhnlicher Krystall. Natürlich können die mimetischen Krystalle auch optisch anomal sein; doch $\mathrm{mu} ß$ dies stets als eine Ausnahme angesehen werden.

II. Die optisch anomalen Krystalle besitzen gewöhnlich einen sehr regelmäßigen Aufbau (wenn die Anomalie nicht mehr zufällig ist, z. B. durch einseitigen Druck hervorgerufen), indem sie aus Pyramiden oder

1) Die bier erwähnten Tatsachen sind wohl alle frïher bekannt; namentlich hat Brauns (Die optischen Anomalien der Krystalle, Leipzig 1891) die meisten erwähnt. Doch meine ich, daß eine solche Zusammenstellung, die nach meinem Wissen noch nicht vorgenommen ist, für das Verstăndnis dieser Phänomene von Bedoutung sein wird.

Groth, Zeitschrift f Krgstallogr. L. 
Sectoren bestehen, die in dem Mittelpunkt oder der Mittellinie des Krystalls zusammenstoßen; die mimetischen Krystalle können auf ähnliche Weise gebaut sein, meistens bestehen sie jedoch zugleich oder allein aus mehr oder weniger regelmäßigen dünnen Lamellen.

III. Hiermit steht in Verbindung, daß die mimetischen Krystalle sehr oft eine Tendenz zur Bildung von secundären $Z$ willingen zeigen; namentlich bei Erwärmung bilden sich oft neue Zwillingslamellen, oder die früheren Grenzen verschieben sich. Bei den optisch anomalen Krystallen kommen solche Phänomene nicht vor.

IV. Wie bekannt, besitzen viele oder vielleicht die meisten mimetischen Krystalle eine bestimmte Umwandlungstemperatur, bei welcher sie in die höher symmetrische Krystallform übergehen, und dieser Übergang ist oft ziemlich plötzlich. Bei den anomalen Krystallen existiert natürlich keine solche dimorphe Umwandlung; übrigens sind in vielen Fällen die Verhältnisse bei Erwärmung nicht bekannt; es scheint jedoch auch hier das gewöhnlichste zu sein (z. B. Apatit, Turmalin und teilweise auch Vesuvian), daß die anomalen Eigenschaften bei höheren Temperaturen weniger hervortretend werden.

V. Wenn die anomalen Krystalle aus Sectoren aufgebaut sind, scheint es, daß die Grenzen zwischen den einzelnen Sectoren nur nach den Krystalllkanten und zugleich nach allen existierenden Kanten gehen; jede Fläche bildet also die Basis einer Pyramide. Wenn die mimetischen Krystalle Sectorenaufbau besitzen, stehen die Grenzen zwischen den einzelnen Individuen in keiner directen Beziehung zu der äußeren Krystallform; wenn der Fall auch eintrifft, daß diese zufälligerweise mit dem inneren Bau übereinstimmen, werden die Zwillingsgrenzen doch nicht genau in den Kanten austreten, sondern meistens mehr oder weniger dicht neben diesen.

Will man hiernach untersuchen, wie sich der Britholith in den genannten Beziehungen verhält, so zeigt es sich sehr schwierig, die verschiedenen Tatsachen zu constatieren, weil die optische Anomalie so schwach hervortretend ist. Was die Inhomogenität betrifft, so ist schon früher darauf aufmerksam gemacht worden, daß einige Krystalle einaxig, andere schwach zweiaxig sind; bei den letzteren scheint auch der Axenwinkel etwas variabel zu sein; die anomalen Krystalle des Britholiths besitzen stets eine ausgeprägte Sectorenstructur ohne Spur von Lamellen; Erwärmungsversuche habe ich des dazu ungeeigneten Materials wegen nicht gemacht. Was die Lage der optischen Grenzlinien angeht, habe ich stets gefunden, daß sie, wo sie überhaupt deutlich sichtbar sind, in den Krystallkanten ausgehen. Nach der Beschreibung von Winther kommt es hier vor, daß in den Ecken des Hexagons (das ja hauptsächlich von den rhombischen Flächen (010) gebildet wird) gewöhnlich ein wenig von den Prismenflächen sich zeigt, 
wodurch ein einspringender Winkel von $1^{0} 47^{\prime} 1$ ) gebildet wird. Dies habe ich nicht so gefunden; alle ein- oder ausspringenden Winkel sind meiner Erfahrung nach durch hypoparallele Verwachsung zustande gekommen, und die oplische Structur wird sehr wenig von dieser beeinflußt.

Soweit man also die undeutlichen optischen Verhältnisse erkennen kann, sprechen diese absolut nicht gegen die hexagonale Krystallform; viele Mineralien, wie z. B. Apatit und Beryll, können auf ganz ähnliche Weise aufgebaut sein mit noch stärker hervortretenden optischen Anomalien und sind doch allgemein als hexagonal anerkannt.

Deutliche Ätzfiguren habe ich nicht hervorbringen können; sie würden natürlich sehr wertvoll für die Bestimmung der Krystallform sein; doch würde man dabei nicht die gewöhnlichste Form (10T0) von der rhombischen (010) unterscheiden können.

Es muß hier noch auf die leichte Löslichkeit in Salzsäure aufmerksam gemacht werden, da diese Eigenschaft für das Bestimmen des Minerals große Bedeutung haben kann. Der Britholith wird von kochender verdünnter Salzsäure so stark angegriffen, daß ein Körnchen von ungefähr $1 \mathrm{~mm}$ Durchmesser schon in ein paar Minuten vollkommen gelöst ist, während das fein pulverisierle Mineral beinahe augenblicklich verschwindet. Vor dem Isütrohr wird der Britholith hellbraun und undurchsichtig, ist aber unschmelzbar; bei noch höherer Temperatur, wie im Lichtbogen, schmilzt er und erstarrt zu einer kugeligen Masse von ungefähr derselben Farbe und demselben Durchsichtigkeitsgrad wie das ursprüngliche Mineral. Diese Masse wird, wie es aus den Dünnschliffen hervorgeht, von einem Individuum gebildet, das mit dem ursprünglichen parallel oder beinahe parallel ist; der neugebildete Krystall ist wie der ursprüngliche einaxig und optisch negativ, die Doppeltbrechung ist aber viel stärker. Nach Messungen mit Compensator, die des ungünstigen Materials wegen nicht sehr genau sein können, ist für den ursprünglichen Britholith:

$$
\omega-\varepsilon=0,004 \text {, }
$$

für den geschmolzenen dagegen:

$$
\omega-\varepsilon=0,03 \text {. }
$$

In Längsschliffen zeigen sich in dem geschmolzenen Britholith deutliche Spaltrisse nach Basis, und auch makroskopisch ist eine solche Spaltbarkeit deutlich sichtbar, von welcher sich an dem ursprünglichen Mineral keine Spur zeigt. Auch sieht man in den Längsschliffen viele recht grobe verticale Linien, die jedoch nicht sowohl auf Spaltbarkeit deuten, sondern vielmehr darauf, daß der Krystall aus Säulen aufgebaut ist, die ein wenig

1) Dieser Winkel sollte übrigens mit den für den rhombischen Britholith angegebenen Axenverhältnissen nicht $1047^{\prime}$, sondern dreimal so groß, also $\mathbf{6}^{0} 21^{\prime}$, sein. 
voneinander abstehen; im Querschliff zeigt sich der ganze Krystall aus kleinen sechseckigen Partien zusammengesetzt, die alle gleich orientiert sind.

Ob diese neugebildete Substanz mit dem Britholith identisch ist, läßt sich schwierig sagen. Das Mineral besteht ja meistens aus recht wenig flüchtigen Elementen, die Zusammensetzung kann also nach dem Schmelzen von der ursprünglichen nicht wesentlich verschieden sein. Es wäre denkbar, daß der Britholith vom ersten Anfang die größere Doppelbrechung gehabt hätte, die nachher verloren gegangen wäre; das Phänomen bei Erhitzung würde dann gewissermaßen mit demjenigen, das der Gadolinit darbietet, vergleichbar sein. Während es ja bei den Mineralien mit seltenen Erden sehr gewöhnlich ist, $\mathrm{daB}$ die ursprünglich anisotrope Substanz amorph geworden ist, kennt man noch kein Beispiel dafür, daß sie eine ziemlich gleichmäßige niedrigere Doppeltbrechung angenommen hat, und es scheint auch recht unwahrscheinlich, daß dies der Fall sein sollte. Man muß dann vielleicht am ehesten annehmen, daß der Britholith dimorph ist mil zwei hexagonalen Modificationen, von welchen die eine bei Erstarrung unter gewöhnlichem Druck gebildet wird, während die andere unter den in der Natur herrschenden Verhältnissen sich bildet. Die beiden Formen würden dann in ungefähr derselben Beziehung zueinander stehen wie Augit und Hornblende. 